CLAWAR 2018: 21st International Conference on Climbing and Walking Robots and the Support Technologies for Mobile Machines, Panama City, Panama, 10-12 September 2018

\title{
A WALKING ASSISTANT USING BRAKES AND LOW COST SENSORS*
}

\author{
LUCIANO CANTELLI, DARIO GUASTELLA，LUCIANO MANGIAMELI, \\ CARMELO DONATO MELITA, GIOVANNI MUSCATO \\ Università degli Studi di Catania Dipartimento di Ingegneria Elettrica Elettronica e \\ Informatica, viale A. Doria 6 \\ CATANIA, 95125, ITALY \\ DOMENICO LONGO \\ Università degli Studi di Catania, Dipartimento di Agricoltura, Alimentazione e \\ Ambiente - Via S. Sofia 100, 95123 CATANIA Italy
}

\begin{abstract}
Smart Walkers are a category of robotic assistive devices particularly useful for the elderly. These systems help the users in locomotion and with respect to the classical walkers, reduce the risk of fall. This work presents a new system that has been developed using low-cost time of flight laser sensors in place of the more expensive laser scanners. Control of direction is performed by guiding the user by mean of brakes on the rear wheels. Other sensors include an inertial measurement unit with magnetometer and an interface with an external absolute localization system. Preliminary trials have been performed on a graphical and physical simulator implemented in the V-Rep framework.
\end{abstract}

\section{Introduction}

\subsection{Assistive mobility devices}

Nowadays it is well known that the proportion of elderly people in the society is constantly growing. As a consequence, it becomes really important to develop assistive systems to allow a better integration in the society of elderly people in their daily activities. Technology can help and several devices have been developed for the so-called Ambient Assistive Living [1]. The European Commission has also started a specific program for AAL [2] and many countries have developed their specific research plans. Recently the International Standard Organisation has developed the standard ISO 13482:2014 which specifies requirements and guidelines for the inherently safe design, protective measures, and information for use of personal care robots.

\footnotetext{
" This work is supported by the project MISE PON 2014-2020 - HORIZON 2020 - "SUMMIT", $\mathrm{F} / 050270 / 01-03 / \mathrm{X} 32$
} 
One of the most important activity that is compromised by age or by some disability, is locomotion. As a consequence, an important function of several devices is to guarantee mobility in autonomy of the users. Walkers are important devices, since consent a physical support and stabilizing effect for those persons with reduced mobility and balance problems. These devices allow also to perform rehabilitation exercises and in most cases are more useful and best accepted with respect to wheelchairs [3].

However, several studies report that the adoption of walkers in many situations does not decrease the probability of falls of the users [4]. Walkers sometime can bump or become entangled into obstacles, or tumble into steps or stairs and as a consequence become the main cause of falls.

\subsection{State of the art on Smart walkers}

Several researchers have studied how to improve a classical walkers introducing some sensors, actuators and control strategies. Such devices are usually known as "smart walkers" and in [5] a review of the functionalities implemented on several systems is presented. In particular devices can be classified with respect to their autonomy, shared-control capability, manual guidance, sit-to-stand help, user's state monitoring. In particular, an important distinction is between motorized walkers and passive walkers. Walkers with active motors connected to the wheels help the user in the mobility, by navigating autonomously in the environment. On the other side passive walkers through the adoption of sensors and sometimes brakes in the wheels, guide the users to their desired route leaving them the responsibility of the motion. Previous work concerning the adoption of brakes for guidance of the trajectory have been reported in [6], where expensive servo brakes and laser scanners are used and in [7], where a detailed study of the trajectory control by brakes is described.

It is important to underline that the functions of a smart walker need to be designed specifically for a precise class of users and that universal solutions do not exists.

\section{The proposed solution}

One of the purpose of the project SUMMIT is to design build and test on real environments an Internet of Things platform based on several smart objects. One of the testing scenario is concerning Smart Health and among the smart objects a smart walker has been selected, to be developed and tested.

The system should have navigation capabilities within a domestic environment. Localization is then performed by interfacing with a specific network in the environment, obstacle avoidance by means of several distance sensors, while inertial sensors monitor bump into obstacles and are be adopted to predict the risk of fall. 
Actuators in the brakes of the rear wheels are installed to guide the users toward desired locations, avoiding obstacles, while a haptic interface in the handlebars sense user's intentions. The main innovative aspect of this work is the adoption of low cost time of flight laser sensors, the integration with an external localization system and the development and implementation of a predictive strategy to prevent the risk of fall.

\subsection{Block diagram of the proposed system}

The draft architecture of the proposed system is reported in Fig. 1. Where all the adopted sensors, actuators and interfaces are reported. In the following sections a short description of the main components is described.

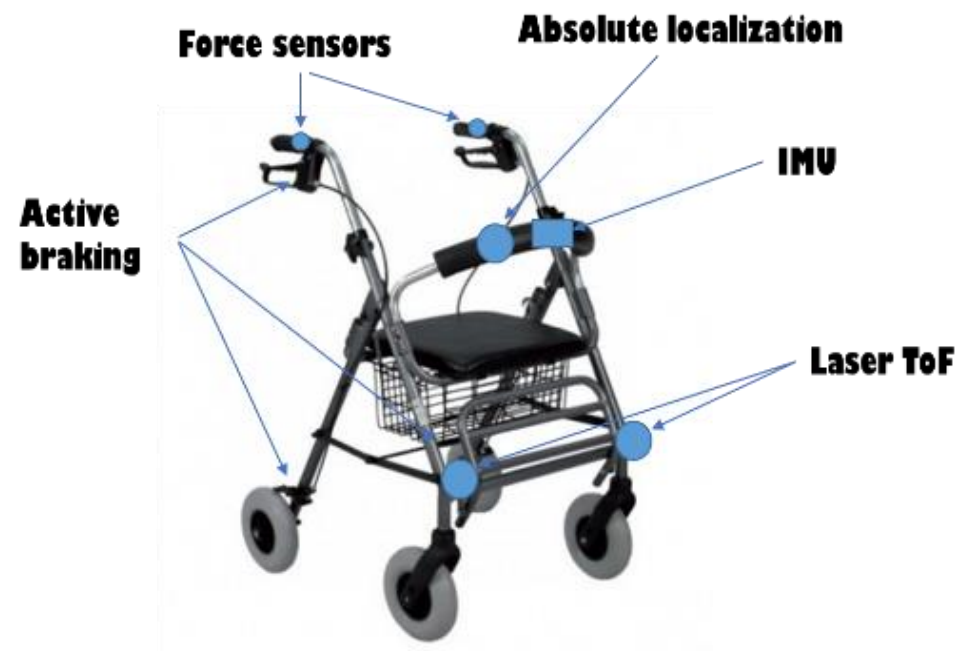

Figure 1. Block diagram of the installed systems on a commercial walker.

\subsection{Time of flight laser sensors}

In order to get a precise obstacle detection without using expensive laser scanner, we decided to adopt some innovative Time-of- Flight (ToF) laser-ranging modules, the VL53L0X by STMicroelectronics. This sensor is housed in a very small package and can measure absolute distances up to $2 \mathrm{~m}$ with a Field Of View of $25^{\circ}$. This distance is considered more than adequate, considering the typical low speeds and the domestic environments.

These sensors can be adopted into three different modalities: Single ranging, Continuous ranging and Timed ranging with three ranging profiles: High speed, High accuracy and Long range.

In particular, we adopted the three sensors integrated into a microcontroller board X-NUCLEO-53L0A1. The system has been also tested mounted on a servo 
motor, thus allowing to get a scan of the environment, as it is shown in Fig.2. An example of a scan is reported in Fig. 3. The time needed for a $360^{\circ}$ scan is of about $2 \mathrm{~s}$, with a resolution of $10^{\circ}$.

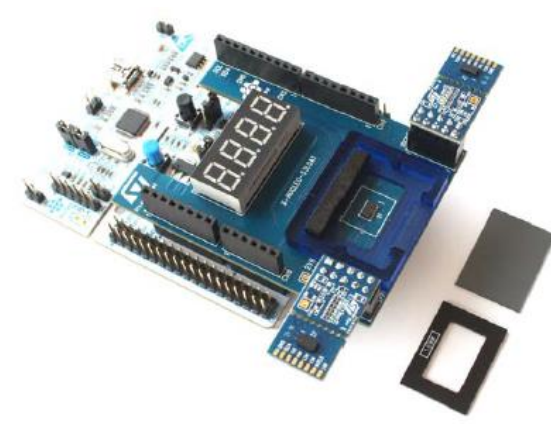

(a)

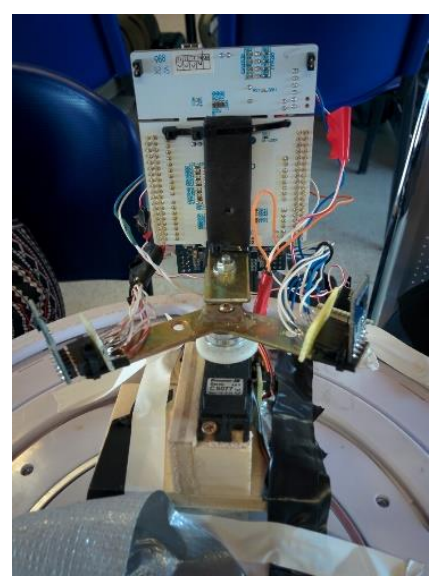

(b)

Figure 2. The evaluation board X-NUCLEO-53L0A1 for the VL53L0X sensors (a) and how it was mounted on a servomotor for environment scanning.

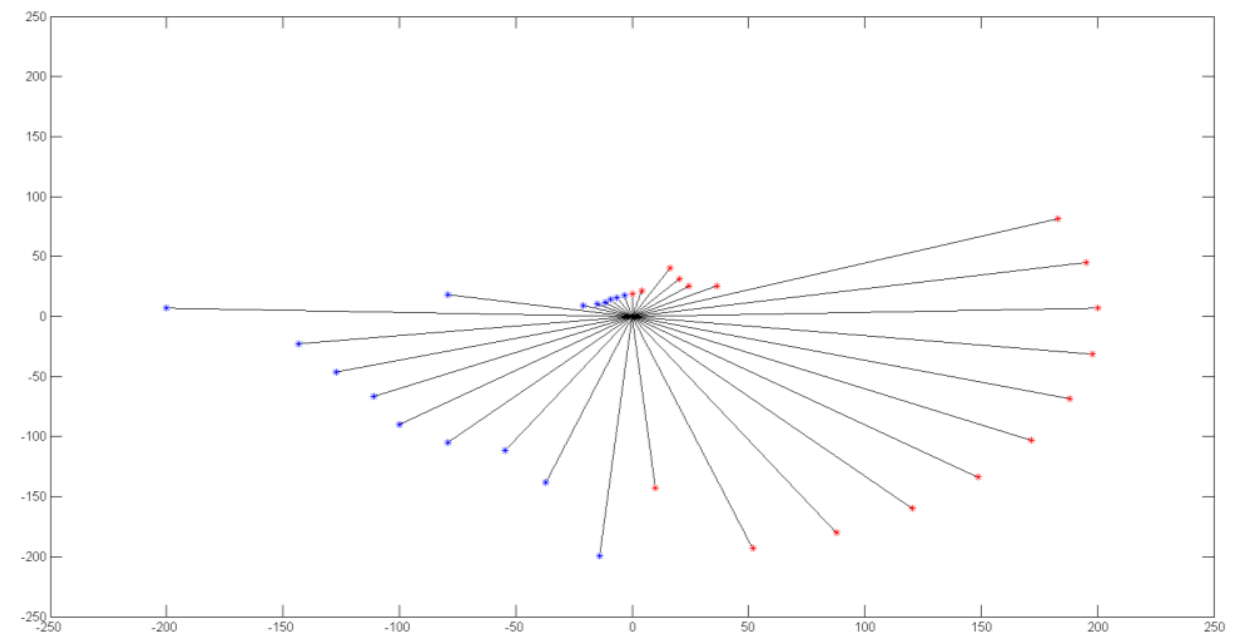

Figure 3. A $360^{\circ}$ scan obtained from the fusion of the information from the three sensors (dimensions in $\mathrm{cm}$.). 


\subsection{Sensor-Tile IMU}

Information on the accelerations of the system and on its orientation is obtained by using the STLCS01V1 SensorTile system by STMicroelectronics (Fig.4).

This is a very compact module which includes a 32bit MCU, a 3D accelerometer and 3d gyroscope, a 3D magnetometer, a pressure sensor, a MEMS microphone, a Bluetooth low energy interface and several other external communication interfaces.

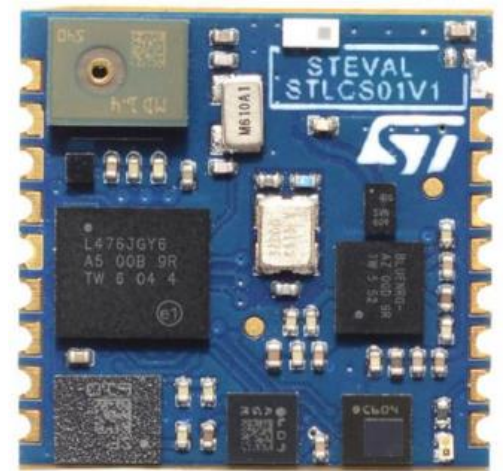

Figure 4. The sensorTile STLCS01V1 board.

In particular, in our application it is adopted for detecting bumps into obstacles and to obtain the absolute orientation of the system.

\subsection{Absolute Localization System}

In order to get absolute localization of the walker in the environment a smart multisensor assistive system has been developed in our Department [8]. This system performs a data fusion algorithm between a wireless sensor network, based on ultrasound sensors for trilateration, and odometry data, gathered by an Inertial Measurement Unit. The average accuracy of the localization in a typical indoor environment is about $3 \mathrm{~cm}$.

\subsection{Obstacle Avoidance Strategy}

As an algorithm to avoid obstacles a potential field approach has been chosen [9]. Data from the map of the environment are fused, by using a Fuzzy Logic algorithm [10], with the Time of Flight sensors to implement a repulsive strategy on the brakes of the walker. Information from the IMU are also adopted to detect bumps and its magnetometers allow the estimation of the orientation of the user. 


\section{Results on simulations}

As a first step, before implementing the strategies on a real hardware, a simulation environment has been designed by using the V-Rep environment. V-Rep is a tool by Coppelia robotic very useful for graphical and physical simulation of robotic systems and can be easily interfaced with external software. In particular, in our application the control strategy has been written in MATLAB. In this case only three fixed Time of flight sensors have been adopted.

In the Figures 5 and 6 some screenshots of the simulations are reported. In particular, in the Figure 6 the graphs on the left lower part show the minimum distance with an obstacle, while on the right upper part, the views of three virtual cameras, placed on the three laser sensors positions, are shown. The three red cones represent the Field Of View of the ToF sensors.

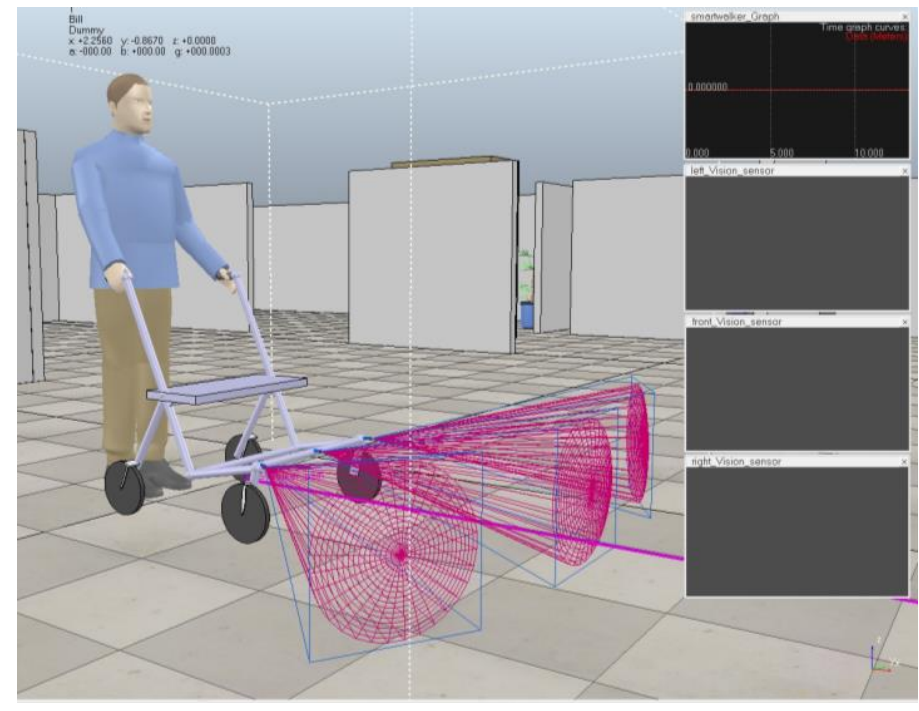

Figure 5. Simulation of the smart walker on a virtual environment in V-Rep. 

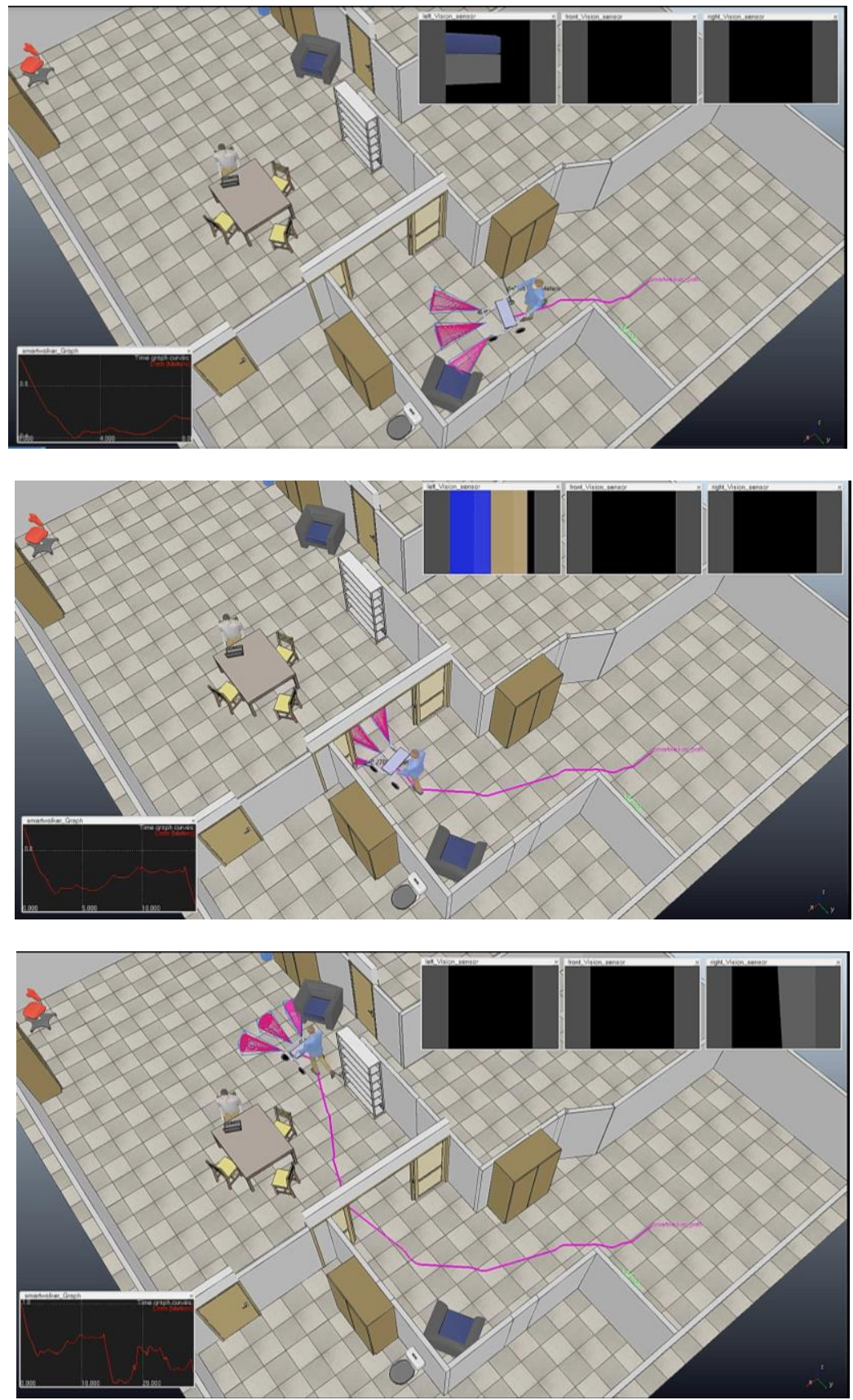

Figure 6. Sequence of a typical path of the smart walker in an indoor domestic environment. 


\section{Conclusions}

This work reported some preliminary results on a new low-cost architecture for a smart walker. The main sensors and the overall strategy has been designed and is under implementation and test on the real hardware. A physical simulator has been implemented and the results are promising. Final implementation and experimental trials on the real system with real users are ongoing.

\section{References}

1. Rashidi, P., \& Mihailidis, A. (2013). A survey on ambient-assisted living tools for older adults. IEEE journal of biomedical and health informatics, 17(3), 579-590.

2. ACTIVE AND ASSISTED LIVING PROGRAMME http://www.aaleurope.eu/

3. Martins, M. M., Santos, C. P., Frizera-Neto, A., \& Ceres, R. (2012). Assistive mobility devices focusing on smart walkers: Classification and review. Robotics and Autonomous Systems, 60(4), 548-562.3.

4. B.A. West, G. Bhat, J. Stevens, G. Bergen, "Assistive devices use and mobility-related factors among adults aged $>=65$ years", Journal of Safety Research, Vol.55, pp. 147-150, 2015.

5. Martins, M., Santos, C., Frizera, A., \& Ceres, R. (2015). A review of the functionalities of smart walkers. Medical engineering and physics, 37(10), 917-928.

6. Hirata, Y., Hara, A., \& Kosuge, K. (2007). Motion control of passive intelligent walker using servo brakes. IEEE Transactions on Robotics, 23(5), 981-990.

7. Fontanelli, D., Giannitrapani, A., Palopoli, L., \& Prattichizzo, D. (2015, December). A passive guidance system for a robotic walking assistant using brakes. In Decision and Control (CDC), 2015 IEEE 54th Annual Conference on (pp. 829-834). IEEE.

8. B. Ando', S. Baglio, C.O. Lombardo, RESIMA: an assistive paradigm to support weak people in indoor environment, IEEE Transactions on Instrumentation and Measurement, vol. 63, no. 11, pp.2522-2528, 2014.

9. Khatib, O. (1986). Real-time obstacle avoidance for manipulators and mobile robots. In Autonomous robot vehicles (pp. 396-404). Springer, New York, NY.

10. G Muscato, Fuzzy control of an underactuated robot with a fuzzy microcontroller, Microprocessors and Microsystems 23 (6), 385-391, 1999. 